\title{
The Riesz Decomposition of Set-Valued Superpramart
}

\author{
Shuyuan Li, Gaoming Li, Hang Dong, Caoshan Wang \\ College of Science, Engineering University of Armed Police Force, Xi'an, China \\ Email:379467329@qq.com
}

Received 10 March 2016; accepted 12 July 2016; published 15 July 2016

\begin{abstract}
The paper proves the convergence theorem of set-valued Superpramart in the sense of weak convergence under the $X^{*}$ separable condition. Using support function and results about real-valued Superpramart, we give the Riesz decomposition of set-valued Superpramart.
\end{abstract}

\section{Keywords}

Set-Valued, Superpramart, Weak Convergence, Riesz Decomposition

\section{Introduction}

Reference [1] gives Riesz decomposition of set-valued supermartingale in real space and promotes the results to reflexive Banach spaces (reference [2]). Reference [2] gives the counter-example that not all of the set-valued martingale has Riesz decomposition in a two-dimensional plane case. The fundamental reason is the defects of algebraic operation on hyperspace. Therefore, the research can pursue the unstrict sense of Riesz decomposition instead of studying various sense of Riesz decomposition. Reference [2] shows the other Riesz decomposition of set-valued supermartingale in real space. Reference [3] gives Riesz decomposition of set-valued supermartingale in the general Banach space under the $X^{*}$ separable condition. References [4] and [5] research Riesz decomposition of set-valued submartingale in the general Banach space. Reference [6] studies Riesz decomposition in weak set-valued Amart. Reference [7]-[9] gives every sense of Riesz decomposition of set-valued Pramart in the general Banach space under the $X^{*}$ separable condition. Reference [10] studies the problems of Riesz decomposition of set-valued Pramart. All of the above studies have given the necessary and sufficient conditions for Riesz decomposition. The research of every sense of Riesz decomposition of set-valued Superpramart is still rare.

The paper firstly demonstrates convergence theorem that set-valued Superpramart is in the sense of weak convergence under the $X^{*}$ separable condition. On this basis, using support function and results about realvalued Superpramart, we give a class of Riesz decomposition of set-valued Superpramart.

\section{Method}

Assume $\left(X\right.$,th) as a separable Banach space, $D_{1}$ is $X$-fan subset of the columns that can be condensed. $X^{*}$ is the dual space $X . X^{*}$ is separable. $D^{*}=\left\{x_{k}^{*} \in X^{*}, k \geq 1\right\}$ is $X^{*}$-fan subset of the columns that can be condensed, remember 


$$
P_{f(c)}(X)=\{A \subset X: \text { AisNon }- \text { empty closed (convex) subset }\}
$$

Any $A, B \in P_{f}(X)$, define

$$
\begin{gathered}
\|A\|=\sup \{\|x\|: x \in A\} \\
S\left(x^{*}, A\right)=\sup \left\{<x^{*}, x>: x \in A\right\}, x^{*} \in X^{*} \\
d(x, A)=\inf \{|x-y|: y \in A\}, x \in X \\
h^{+}(A, B)=\sup \{d(x, B), x \in A\} \quad h^{-}(A, B)=h^{+}(B, A) \\
h(A, B)=\max \left\{h^{-}(A, B), h^{+}(B, A)\right\}
\end{gathered}
$$

$\left\{A_{n}, n \geq 1, A\right\} \subset P_{f}(X)$ If for any $x^{*} \in X^{*}, \lim S\left(x^{*}, A_{n}\right)=S\left(x^{*}, A\right)$, we call $A_{n}$ weak convergence in $A$, denote as

$$
\begin{gathered}
A_{n} \stackrel{w}{\longrightarrow} A, \text { or }(\mathrm{w}) \lim _{\mathrm{n}} \mathrm{A}_{n}=A . \\
w-\varlimsup_{n} A_{n}=\left\{x \in X: \exists x_{k} \in A_{n_{k}}, k \geq 1, k \rightarrow+\infty, \quad x_{k} \stackrel{w}{\rightarrow} x\right\} \\
s-\frac{\lim }{n} A_{n}=\left\{x \in X: \lim _{n} d\left(x, A_{n}\right)=0\right\}
\end{gathered}
$$

If $s-\frac{\lim }{n} A_{n}=w-\varlimsup_{n} A_{n}=A$, then we call $\left\{A_{n}, n \geq 1\right\}$ Kuratowski-Mosco significance of convergence in A, denote as (K-M) $\lim _{n} A_{n}=A$, or $A_{n} \stackrel{K-M}{\rightarrow} A$.

Assume $(\Omega, \mathcal{G}, \mathrm{P})$ is a complete probability space. $\left\{\mathcal{G}_{n}, n \geq 1\right\}$ is the $\mathcal{G}$ 's rise $\sigma$, and $\mathcal{G}=\vee \mathcal{G}_{n}, \bar{T}(T)$ indicates stop (bounded stopping), $T(\sigma)=\{\tau \in T ; \tau \geq \sigma\} . \sigma \in T$, said the set value is mapped $\mathrm{F}: \Omega \rightarrow \mathrm{P}_{\mathrm{f}}(\mathrm{X})$ to a random set (or on $\mathrm{G}$ measurable). If for any open set $G$, it has $F^{-}(G)=\{\omega: F(\omega) \cap G \neq \Phi\} \in \mathcal{G}$. We define $\left\{F_{n}, \mathcal{G}_{n}, \mathrm{n} \geq 1\right\}$ as adapted random set columns. If $\forall n \geq 1, F_{n}$ can be measured by $\mathcal{G}_{n}$. If $\int_{\Omega}\|F\| d P<\infty$, F is bounded integrable. $L_{f c}^{1}[\Omega, X]$ represents that the value of $P_{f c}(X)$ is all the integrable bounded random set.

In order to write simply, often eliminating the almost certainly established under the meaning of the equations, inequalities and tag contain relations sense "a.s.", $\left\{F_{n}, \mathcal{G}_{n}, n \geq 1\right\}$. $\left\{x_{n}, \mathcal{G}_{n}, n \geq 1\right\}$ is often referred as $\left\{F_{n}, n \geq 1\right\}$, $\left\{x_{n}, n \geq 1\right\}$.

Definition 1 Supposing $\left\{x_{n}, n \geq 1\right\}$ is a real-valued integrable adapted column

1) If $\forall \varepsilon>0, \lim _{\sigma \in T} \sup _{\tau \in T(\sigma)} P\left\{\omega:\left[x_{\sigma}-E\left(x_{\tau} \mid \mathcal{G}_{\sigma}\right)\right]>\varepsilon\right\}=0$, call $\left\{x_{n}, n \geq 1\right\}$ as Subpramart.

2) If $\forall \varepsilon>0, \lim _{\sigma \in T} \sup _{\tau \in T(\sigma)} P\left\{\omega:\left[x_{\sigma}-E\left(x_{\tau} \mid \mathcal{G}_{\sigma}\right)\right]^{-}>\varepsilon\right\}=0$, call $\left\{x_{n}, n \geq 1\right\}$ as Superpramart.

Definition 2 Supposing $\left\{F_{n}, n \geq 1\right\}$ is a $L_{f c}^{1}[\Omega, X]$ valued adapted random set column

If $\forall \varepsilon>0, \lim _{\sigma \in T} \sup _{\tau \in T(\sigma)} P\left\{\omega: h^{-}\left(F_{\sigma}, E\left(F_{\tau} \mid \mathcal{G}_{\sigma}\right)\right)>\varepsilon\right\}=0$, call $\left\{F_{n}, n \geq 1\right\}$ as set-valued Superpramart.

Definition 3 Supposing $A, B \in P_{f c}(X)$, we call A and B are homothetic, if $C \in P_{f c}(X)$ exists, then it has $A=B+C$.

Definition 4 We call set-valued Superpramart $\left\{F_{n}, n \geq 1\right\}$ has Riesz decomposition, if set-valued martingale $\left\{G_{n}, n \geq 1\right\} \subset L_{f c}^{1}[\Omega, X]$ and set-valued Superpramart $\left\{Z_{n}, n \geq 1\right\} \subset L_{f c}^{1}[\Omega, X]$ exist, $Z \stackrel{K-M}{\longrightarrow}\{0\},(n \rightarrow \infty)$ lets

$$
F_{n}=G_{n}+Z_{n}, n \geq 1
$$

Lemma 1 [11] If $\left\{F_{n}, n \geq 1\right\}$ is set-valued Superpramart, then

1) $\forall x^{*} \in X^{*},\left\{S\left(x^{*}, F_{n}\right), n \geq 1\right\}$ is real-valued Superpramart.

2) $\forall x \in X,\left\{d\left(x, F_{n}\right), n \geq 1\right\}$ is real-valued Subpramart.

Lemma 2 Supposing $\left\{x_{n}, n \geq 1\right\}$ is real-valued Superpramart and $\frac{\lim E}{\tau}\left|x_{\tau}\right|<\infty$, then $\lim _{n} x_{n}$ exist and is 
integrable.

Proof: $\left\{-x_{n}, n \geq 1\right\}$ is the real-valued Subpramart, and reference [12] theorem 5 (corollary 1) is known.

Lemma 3 [7] Supposing $\left\{A_{n}, n \geq 1\right\} \subset P_{f c}(X)$, if

1) $\sup \left\|A_{n}\right\|<\infty$;

2) $\forall x^{*} \in D^{*}, \lim _{n} S\left(x^{*}, A_{n}\right)$ are limited existing, if it has $A \in P_{f_{c}}(X)$, let $A_{n} \stackrel{w}{\longrightarrow} A$.

Lemma 4 Supposing $\left\{F_{n}, n \geq 1\right\} \subset L_{f c}^{1}(X)$ is set-valued Superpramart and $\sup _{\tau \in T} E\left\|F_{\tau}\right\|<\infty$, then it has random set $F \in P_{f c}(X)$, let $F_{n} \stackrel{w}{\longrightarrow} F$.

Proof: $\forall x^{*} \in X^{*}$, we know $\left\{S\left(x^{*}, F_{n}\right), n \geq 1\right\}$ is a real-valued Superpramart from reference [11] theorem 3.1, and because $\frac{\lim }{T} E\left|\sigma\left(x^{*}, F_{\tau}\right)\right| \leq\left\|x^{*}\right\| \frac{\lim }{T} E\left\|F_{\tau}\right\|<\infty$, we know $\lim _{n} S\left(x^{*}, F_{n}\right)$ exists and is limited from reference [12] theorem 5, and through the list of $D^{*}$, we know $\lim S\left(x^{*}, F_{n}\right)$ exists and is limited in little-known set of $\mathrm{N}, \omega \in \Omega \backslash N, x^{*} \in D^{*}$, by the maximum inequality and Lemma 3 , the existence of $\mathrm{F}$ lets

$\lim S\left(x^{*}, F_{n}\right)=S\left(x^{*}, F\right), \omega \in \Omega \backslash N, x^{*} \in X^{*}$, then by reference [2] corollary 2.1.1 and theorem 2.1.19, we know $F$ is a random set, the conclusion is proved.

Lemma 5 Supposing $\left\{x_{n}, n \geq 1\right\}$ is a real-valued Superpramart, and $\frac{\lim E}{n}\left\|F_{n}\right\|<\infty$, then it has an unique factorization $x_{n}=y_{n}+z_{n}, n \geq 1$, where $\left\{y_{n}, n \geq 1\right\}$ is a real-valued martingale, $\left\{z_{n}, n \geq 1\right\}$ is a real-valued Superpramart, and $\left|z_{n}\right| \rightarrow 0,(n \rightarrow \infty)$.

Proof: From Lemma 2, we know $\lim x_{n}=x \in \mathrm{L}^{1}$, noting $y_{n}=E\left(x \mid \mathcal{G}_{n}\right)$, it's easy to find taht $\left\{y_{n}, n \geq 1\right\}$ is a real-valued martingale, making $z_{n}=\stackrel{n}{x}_{n}-y_{n}, n \geq 1$, it's easy to know $\left\{z_{n}, n \geq 1\right\}$ is a real-valued Superpramart, and $\lim _{n} z_{n}=\lim _{n} x_{n}-\lim _{n} E\left(x \mid \mathrm{B}_{\mathrm{n}}\right)=0$, it also has $\left|z_{n}\right| \rightarrow 0,(n \rightarrow \infty)$.

The uniqueness is proved by the following: Supposing $x_{n}=y_{n}^{(i)}+z_{n}^{(i)}, n \geq 1, i=1,2$, so $y_{n}^{(1)}-y_{n}^{(2)}=z_{n}^{(2)}-z_{n}^{(1)}, n \geq 1$, because $\left\{y_{n}^{(i)}, n \geq 1\right\}(i=1,2)$ is a real-valued martingale, $\left\{z_{n}^{(2)}-z_{n}^{(1)}, n \geq 1\right\}$ is a real-valued martingale from the above equation, then

$\left|y_{n}^{(1)}-y_{n}^{(2)}\right|=\underline{\varliminf_{k}}\left|E\left(y_{n+k}^{(1)}-y_{n+k}^{(2)} \mid \mathcal{G}_{n}\right)\right| \leq \underline{\underline{\lim }} E\left(\mid z_{n+k}^{(2)}-z_{n+k}^{(1)} \| \mathcal{G}_{n}\right)=0$, (from reference [12] theorem 7), so $y_{n}^{(1)}=y_{n}^{(2)}, z_{n}^{(2)}=z_{n}^{(1)}, n \geq 1$, the uniqueness is proved. Lemma 6 Supposing $\left\{F_{n}, n \geq 1\right\} \subset L_{f c}^{1}(X)$ is a set-valued Superpramart, and $\sup _{\tau \in T} E\left\|F_{\tau}\right\|<\infty$, if
$s-\underline{\lim } F_{n}=F$, then $F_{n} \stackrel{\text { if }}{\longrightarrow} F$.

Prßof: From Lemma 4, we know the random set $G \in P_{f c}(X)$ exists, then it has $F_{n} \stackrel{w}{\longrightarrow} G$, and

$$
d\left(x, F_{n}\right)=\sup _{i}\left[<x_{i}^{*}, x>-S\left(x_{i}^{*}, F_{n}\right)\right] x_{i}^{*} \in D^{*}
$$

noting $y_{n}^{i}=<x_{i}^{*}, x>-S\left(x_{i}^{*}, F_{n}\right)$, from reference [11] theorem 3.1, we know $\left\{y_{n}^{i}, n \geq 1\right\}, i \geq 1$ is a real-valued consistent Subpramart, then from reference [2] Lemma 4.4.2, we know the little-known set $N$ exists, $\lim _{n} d\left(x, F_{n}\right)=\sup _{i}\left(\lim _{n} y_{n}^{i}\right)=\sup _{i}\left[<x_{i}^{*}, x>-S\left(x_{i}^{*}, G\right)\right]=d(x, G), \forall x \in D_{1}, \omega \in \Omega \backslash N$, From inequality $|d(x, A)-d(y, A)| \leq\|x-y\|$ and the usual density method, we known $\lim _{n} d\left(x, F_{n}\right)=d(x, G), \forall x \in X$, $\omega \in \Omega \backslash N$, it indicates $s-\underline{\lim } F_{n}=G$, then $F_{n} \stackrel{w}{\longrightarrow} F$.

Lemma 7 Supposing $\left\{\widehat{F_{n}, n} \geq 1\right\} \subset L_{f_{c}}^{1}(X)$ is a set-valued Superpramart, and $\sup _{\tau \in T} E\left\|F_{\tau}\right\|<\infty$, the followings are equivalent:

1) $\left\{F_{n}, n \geq 1\right\}$ can be the Riesz decomposition.

2) $\forall n \geq 1, F_{n}$ and $E\left(F \mid \mathcal{G}_{n}\right)(\mathrm{n} \geq 1)$ are homothetic, where $F_{n} \stackrel{w}{\longrightarrow} F$.

Proof: We prove $F \in L_{f c}^{1}[\Omega, X]$ firstly, because $\left\|F_{n}\right\|=\sup _{i} S\left(x_{i}^{*}, F_{n}\right)$, it's easy to know

$\lim _{n}\left\|F_{n}\right\| \geq \sup _{i}\left[\lim _{n} S\left(x_{i}^{*}, F_{n}\right)\right]=\sup _{i} S\left(x_{i}^{*}, F\right)=\|F\|$, by the lemma Fatou, we know

$\int_{\Omega}\|F\| d P \leq \int_{\Omega} \frac{\lim }{n}\left\|F_{n}\right\| d p \leq \frac{\lim }{n} \int_{\Omega}\left\|F_{n}\right\| d P \leq \frac{\lim }{n} E\left\|F_{n}\right\|<\infty$, then $F \in L_{f c}^{1}[\Omega, X]$.

$1) \Rightarrow 2$ ) because $F_{n_{*}}=G_{n}+Z_{n_{*}}, Z_{n} \stackrel{K-M}{\longrightarrow}\{0\},(n \rightarrow \infty)$,

Then, $S\left(x^{*}, F_{n}\right)=S\left(x^{*}, G_{n}\right)+S\left(x^{*}, Z_{n}\right)$. 
From Lemma 1, Lemma 6 and reference [2] lemma 4.1.3, it's easy to know the above equation is the Riesz decomposition of real-valued Superpramart $\left\{S\left(x^{*}, F_{n}\right), n \geq 1\right\}$, and from Lemma 5 and its proof process, we notice $\lim S\left(x^{*}, F_{n}\right)=S\left(x^{*}, F\right) . x^{*} \in X^{*}$ and know $S\left(x^{*}, G_{n}\right)=E\left(S\left(x^{*}, F\right) \mid \mathcal{G}_{n}\right)=S\left(x^{*}, E\left(F \mid G_{n}\right)\right)$, by the separability of $X^{*}$ and the continuity of $X^{*}$ support function, we know from reference [2] corollary 1.4.1 that: $G_{n}=E\left(F \mid \mathcal{G}_{n}\right)$, namely, $F_{n}$ and $E\left(F \mid \mathcal{G}_{n}\right)$ are homothetic.

$2) \Rightarrow 1)$ Noting $G_{n}=E\left(F \mid \mathcal{G}_{n}\right)$, it's easy to know $\left\{G_{n}, n \geq 1\right\}$ is the value martingale of $L_{f c}^{1}[\Omega, X]$, and $\sup E\left\|G_{n}\right\| \leq E\|F\|<\infty$, making $F_{n}=G_{n}+Z_{n}$, the following is the proof that $\left\{Z_{n}, n \geq 1\right\}$ is the value Superpramart of $L_{f c}^{1}[\Omega, X]$, because

$$
\begin{aligned}
& S\left(x^{*}, F_{n}\right)=S\left(x^{*}, G_{n}\right)+S\left(x^{*}, Z_{n}\right) \\
& S\left(x^{*}, Z_{n}\right)=S\left(x^{*}, F_{n}\right)-S\left(x^{*}, G_{n}\right)
\end{aligned}
$$

It's easy to prove $\left\|Z_{n}\right\| \leq\left\|F_{n}\right\|+\left\|G_{n}\right\|$, so $E\left\|Z_{n}\right\| \leq E\left\|G_{n}\right\|+E\left\|F_{n}\right\|<\infty$.

$E\left(F_{t} \mid \mathcal{G}_{\sigma}\right)=G_{\sigma}+E\left(Z_{t} \mid \mathcal{G}_{\sigma}\right), \sigma \in T, \tau \in T(\sigma)$, from reference [2] lemma 5.3.6, we know

$$
\begin{aligned}
& H^{+}\left(E\left(Z_{\tau} \mid \mathcal{G}_{\sigma}\right), Z_{\sigma}\right) \\
& =\sup _{i}\left[-S\left(x_{i}^{*}, Z_{\sigma}\right)+S\left(x_{i}^{*}, E\left(Z_{\tau} \mid \mathcal{G}_{\sigma}\right)\right)\right], x_{i}^{*} \in D^{*} \\
& =\sup _{i}\left[-S\left(x_{i}^{*}, F_{\sigma}\right)+S\left(x_{i}^{*}, G_{\sigma}\right)+S\left(x_{i}^{*}, E\left(F_{\tau} \mid \mathcal{G}_{\sigma}\right)\right)-S\left(x_{i}^{*}, G_{\sigma}\right)\right], x_{i}^{*} \in D^{*} \\
& =\sup _{i}\left[-S\left(x_{i}^{*}, F_{\sigma}\right)+S\left(x_{i}^{*}, E\left(F_{\tau} \mid \mathcal{G}_{\sigma}\right)\right]\right. \\
& =h^{+}\left(E\left(F_{\tau} \mid \mathcal{G}_{\sigma}\right), F_{\sigma}\right)
\end{aligned}
$$

Then, we know $\left\{Z_{n}, n \geq 1\right\}$ is set-valued Superpramart the proof is set below $Z_{n} \stackrel{K-M}{\longrightarrow}\{0\},(n \rightarrow \infty)$, because

$S\left(x_{i}^{*}, Z_{n}\right)=S\left(x_{i}^{*}, F_{n}\right)-S\left(x_{i}^{*}, G_{n}\right), x_{i}^{*} \in D^{*}$

$=S\left(x_{i}^{*}, F_{n}\right)-S\left(x_{i}^{*}, E\left(F \mid \mathcal{G}_{n}\right)\right)$

$=S\left(x_{i}^{*}, F_{n}\right)-E\left(S\left(x_{i}^{*}, F\right) \mid \mathcal{G}_{n}\right)$, and from the list of $D^{*}$, we know the little-known set $N_{1}$, and $\lim _{i} S\left(x^{*}, Z_{n}\right)=0$, $\omega \in \Omega \backslash N_{1}, \quad x^{*} \in D^{*}$, using Lemma 3, we know $\lim _{n} S\left(x^{*}, Z_{n}\right)=S\left(x^{*},\{0\}\right), \omega \in \Omega \backslash N_{1}, x^{*} \in X^{*},{ }^{n}$

$$
d\left(x, Z_{n}\right)=\sup _{i}\left[<x_{i}^{*}, x>-S\left(x_{i}^{*}, Z_{n}\right)\right] \quad x_{i}^{*} \in D^{*}
$$

Noting $y_{n}^{i}=<x_{i}^{*}, x>-S\left(x_{i}^{*}, Z_{n}\right)$, from reference [11] lemma 3.2, we know $\left\{y_{n}^{i}, n \geq 1\right\}, i \geq 1$ is a real-valued consistent Subpramart, and from reference [2] lemma 4.4.2, we know the little-known set $N_{2}$ exists, $\lim _{n} d\left(x, Z_{n}\right)=\sup _{i}\left(\lim _{n} y_{n}^{i}\right)=\sup _{i}\left[<x_{i}^{*}, x>-S\left(x_{i}^{*},\{0\}\right)\right]=d(x,\{0\}), \forall x \in D_{1}, \omega \in \Omega \backslash N_{2}$, from inequality $|d(x, A)-d(y, A)| \leq\|x-y\|$ and the usual density method, we know $\lim _{n} d\left(x, F_{n}\right)=d(x,\{0\}), \forall x \in X$, $\omega \in \Omega \backslash N_{2}$, then from reference [2] lemma 4.5.4, $Z_{n} \stackrel{K-M}{\longrightarrow}\{0\},\left(n \rightarrow \rightarrow^{n} \infty\right)$.

\section{Conclusion}

The paper proves the convergence theorem of Superpramart in the sense of weak convergence. And on the basis of this certificate, through the support function and the results of real-valued Superpramart, we give the one of Riesz decomposition forms of set-valued Superpramart. It provides new ideas for the research of Riesz decomposition.

\section{References}

[1] Zhang, W.X. and Gao, Y. (1992) Convergence and Riesz Decomposition of Set-Valued Martingale. Journal of Mathematics, 35, 112-120. (In Chinese)

[2] Zhang, W.X., Wang, Z.P. and Gao, Y. (1996) Set-Valued Stochastic Process. Science Press, Beijing. (In Chinese)

[3] Li, G.M. (2009) A Note on the Set-Valued Martingale Decomposition. Pure Mathematics and Applied Mathematics, 25, 69-71. (In Chinese)

[4] Li, G.M. (2010) A Note on the Riesz Decomposition of Set-Valued Martingale. Fuzzy Systems and Mathematics, 24, 
110-114. (In Chinese)

[5] Li, G.M. and Li, H.P. (2011) A Class of Riesz Decomposition of Set-Valued Martingale. Journal of Jilin University (Science Edition), 49, 1039-1043. (In Chinese)

[6] Liu, C.Y., Li, S.K. and Zhou, H.R. (2002) The Riesz Decomposition of Weak Set-Valued Amart. Applied Probability Statistics, 18, 173-175. (In Chinese)

[7] Li, G.M. (2007) Martingale Decomposition of Set-Valued Premart. Pure Mathematics and Applied Mathematics, 23, 299-303. (In Chinese)

[8] Li, G.M. (2009) The Riesz Approximation of Set-Valued Pramart. Journal of Jilin University, 47, 45-49. (In Chinese)

[9] Li, G.M. (2009) The Riesz Decomposition Theorem of Set-Valued Pramart. Journal of Engineering Mathematics, 26, 377-380. (In Chinese)

[10] Li, G.M. (2011) Another Class of Riesz Approximation of Set-Valued Subpramart. Fuzzy Systems and Mathematics, 25, 109-112. (In Chinese)

[11] Gao, Y. and Zhang, W.X. (1993) Some Results of Set-Valued Pramart. Applied Probability Statistics, 9, 189-197. (In Chinese)

[12] Wang, Z.P. (1986) Another Class of Convergence Theorem of Martingale Sequences. Applied Probability Statistics, 2, 241-246. (In Chinese)

\section{Submit or recommend next manuscript to SCIRP and we will provide best service for you:}

Accepting pre-submission inquiries through Email, Facebook, LinkedIn, Twitter, etc.

A wide selection of journals (inclusive of 9 subjects, more than 200 journals)

Providing 24-hour high-quality service

User-friendly online submission system

Fair and swift peer-review system

Efficient typesetting and proofreading procedure

Display of the result of downloads and visits, as well as the number of cited articles

Maximum dissemination of your research work

Submit your manuscript at: http://papersubmission.scirp.org/ 